\title{
Virtual Screening of 2-hydroxy-1,4-naphthoquinone Derivatives as Antimitotic Agent using Molegro Virtual Docker on Polo Like Kinase 1
}

\author{
Susi Kusumaningrum ${ }^{1,2^{*}}$, Soleh Kosela ${ }^{1}$, Wahono Sumaryono ${ }^{2}$, Emil Budianto ${ }^{1}$, Alfan Danny Arbianto ${ }^{2}$ \\ ${ }^{I}$ Chemistry Department, Faculty of Mathematics and Natural Science, Universiy of Indonesia. \\ ${ }^{2}$ Center of Pharmaceutical and Medical Technology, Agency for Assessment and Application of Technology, Indonesia.
}

Received: 13 October 2014 / Accepted: 30 November 2014

\begin{abstract}
:
Naphthoquinone is one of secondary metabolites that are widespread in nature and found in large amounts [1]. Naphthoquinones are clinically important antitumor drugs containing a quinone moiety, such as anthracyclines, mitoxantrones and saintopin, show excellent anticancer activity [2]. In the previouse studies, Polo like kinase 1 (Plk1) is the preferential target for inhibition of mitotic processing and hence can be chosen as drug target for the treatment of cancer [3]. The aim of the study is lead finding of potential plk1 inhibitor from naphthoquinone derivatives through binding free energy analysis into plk1 using molecular docking. We conducted docking simulation to 50 naphthoquinone derivatives as ligands into plk1 as receptor. The 3D structure of plk1 was downloaded from PDB (PDB ID 3THB). Docking process, the interaction and binding of ligands-protein was done and visualized using Molegro Virtual Docking (MVD). The result showed the predicted docking energy and contact residue which reflected the binding affinities. Somenaphthoquinone derivatives showed reasonably low internal energy binding into Plk1 indicating the docked conformation of the ligands were in their most favorable conformations. It is predicted that naphthoquinone derivative has potency as lead compound to find a new antimitotic candidates for possible therapeutic agents.
\end{abstract}

Key words: Antimitotic agent, Molegro Virtual Docker, naphthoquinone, Polo like kinase 1

\section{Introduction}

Some of naphthoquinone derivatives have been synthesized and evaluate in order to discover more potent anticancer agent. Naphthoquinones derivatives are clinically important antitumor drugs containing a quinone moiety, such as anthracyclines, mitoxantrones and saintopin, show excellent anticancer activity $[1,2,3$, 4]. The National Cancer Institute (NCI), Bethesda, USA, is still playing an articular role in this field and identified that the quinone as an important pharmacophore for anticancer activity.

Antimitotic form is the basis of therapy for patients with multiple types of solid tumors and hematologic malignancies (cancer). An ideal next generation antimitotic target is Polo-like kinase 1 (Plk1) [5, 6, 7].

Currently, molecular docking approach has been used in modern drug design to understand drug-receptor interactions. According to the literature, computational techniques can support and help in making drug design. In addition, it can be used to know mechanism of drugreceptor interactions. In this study we docked polo like kinase 1 with naphthoquinone derivatives by Molegro Virtual Docker (MVD) [8].
This paper report screening of various naphthoquinone derivatives possessing benzolactam moiety bound directly or indirectly to ring system against Plk1 enzyme extracted from Protein Data Bank, by utilizing the Molegro Virtual Docker Software. Various molecular structures of the ligands were docked and scored to identify the ligands that bind similar to reference ligand binding for Plk1 and to estimate the ligands binding affinity for its target.

\section{Experimental}

Hardware and software

Molegro Virtual Docker 6.0 2013 and Marvin Sketch Product version 6.2.3 2013 were used for molecular modeling with Intel ${ }^{\circledR}$ Core $^{\mathrm{TM}}$ i7-3770 processor.

\section{Molecular structure and optimization}

The structure of naphthoquinone derivatives was drawn by Marvin Sketch Product version 6.2.3 2013. The structure of polo like kinase 1 receptor (PDB ID 3THB) was obtained from the Protein Data Bank (http://www.rcsb.org).

\footnotetext{
*Corresponding author: Susi Kusumaningrum,

E-mail: susi.kusumaningrum@gmail.com.
} 
Docking of 2-hydroxy-1,4 naphthoquinone (2HN), 18 derivates of $2 \mathrm{HN}$, doxorubicin and poloxin against Plk1 have performed using MVD software. A single rigid crystal structure of an enzyme is used for docking studies from the 53 crystal structures of Plk1 held in the Protein Data Bank (PDB) [10] accessed at the URL (http://www.rscb.org/pdb) under the criteria that they had reasonable resolution $(\leqslant 2.8 \AA)$ and involved the non-mutated pololike kinase enzyme from Homo sapiens, in the complex with small molecule ligands.

Ten independent runs were performed with the guided differential evolution algorithm, with each of these docking runs returning one solution (pose). The Moldock scoring function used by MVD is derived from the PLP scoring functions originally proposed by Gehlhaar et al and extended later by Yang et al [8].

The 10 solutions obtained from the 10 independent docking runs were re-ranked, in order to further increase the docking accuracy, by using a more complex scoring function. In the MVD, along with the docking scoring terms, a Lennard Jones 12-6 potential and $\mathrm{sp}^{2}$-sp $\mathrm{sp}^{2}$ torsion terms were also used [8].

On the basis of pilot docking studies, the MolDock rerank scores were selected for ranking the inhibitor poses, and for all the pololike kinase 1 docking performed here, the poses selected as the best were taken as those with the highest MolDock re-rank score.

In order to make accurate predictions, it is important that the imported structures have been properly prepared, that is, the atom connectivity and bond orders are correct and partial atomic charges are assigned. PDB files often have poor or missing assignment of explicit hydrogens, and the PDB file format cannot accommodate bond order information. All necessary valence checks and $\mathrm{H}$ atom addition were thus performed using the utilities provided in MVD. The binding site specifies the region of interest where the docking procedure will look for promising poses (ligand conformations).

\section{Results and Discussion}

Molecular docking is an optimization problem, where the objective is to find the ligand binding mode with lowest potential energy. The process of docking involves sampling the coordinate space of the target binding site and scoring each possible ligand pose within that site, the highest scoring pose then taken as the predicted binding mode for that compound. There are many different docking programs now available and they differ in the nature of the sampling algorithms they employ, in their manner of handling ligand and protein flexibility, in the scoring functions they use, and in the cpu time they required. In the studies reported here, MVD was used, because it showed higher docking accuracy when benchmarked against other available docking programs (MD: 87\%, Glide: 82\%, Surflex: 75\%, FlexX: 58\%) and has been shown to be successful in several recent studies, but also for reasons of cost and user-friendliness [9].

MVD automatically identifies potential binding sites (also referred as cavities or active sites) by using its cavity detection algorithm. The cavities within a $30 \times 30$ $\times 30 \AA^{3}$ cube centered at the experimentally known ligand position were used. The cavities that are identified by the cavity detection algorithm are then used by the guided differential evolution search algorithm to focus the search, to that specific area during the docking simulation. In the case of the crystal structures for pololike kinase 1 complexes, the program generally identified five different binding sites (Figure 1). From these five predicted cavities the one with the highest volume is $159.744 \AA$. Duffey was selected for consideration, as it includes the bound ligand [10]. The best studied member of the Plk family is Plk1, which has been implicated in various essential cell-cycle-related processes including centrosome maturation, mitotic entry, checkpoint recovery, spindle assembly, sister chromatid, separation and cytokinesis [11].

One application of molecular docking is to design pharmaceutical in silico by optimizing targeted lead candidates against protein. The lead candidates can be found using a docking algorithm that aims to identify the optimal binding mode of a small molecule (ligand) to the active site of macromolecular target.

Validation of docking was used to ensure orientation and position of ligand binding that was obtained from docking studies by MVD program with ten replication of each running. And then the parameters must be validated in crystal structure of receptor (PDB ID 3THB). Native ligand 3THB in polo like kinase 1 was found in complex with ligand-receptor conformation. After that, it was extracted and put it into appropriate active site for ligand binding to determine the ability of orientation and position of ligand that was observed in cavity ligandreceptor crystal structure. The results, indicate that control docking has optimal orientation of ligand complex binding by Molegro Virtual Docker program, it was able to determine toward active $3 \mathrm{THB}$ which consist of 10 atoms (Figure 1), in cavity 2 (154.624 volumes) and use protein $3 \mathrm{THB}$ of polo like kinase 1 structure crystalline. The methods can be said valid if RMSD of parameters docking showed minimal value. The RMSD of this method was 0.66 .

Nineteen naphthoquinone compounds have been designed into oxime derivatives to obtain more potent compounds as inhibitors of Plk1. The compound is positioned as a ligand or inhibitors that inhibit the receptor or enzyme's performance Furthermore docking of these ligand, doxorubicin, benzolactam and poloxin were performed with the crystal structure of Plk1 and each ligand chosen the best position to determine the rerank score. In each docking run, the best poses were selected on the basis of their MVD re-rank scores and 
the mean of the 5 re-rank scores was then computed as the final score for each compound. The MVD score and the re-rank scores of the best poses for each of the docking studies of naphthoqunone ligand, doxorubicin and benzolactam with polo like kinase 1 are summarized in Table 1. The structure of $2 \mathrm{HN}$ derivatives, doxorubicine, benzolactam and poloxin are showed in Table 2 and Fig.2. From the results of the docking score is known or assumed values as binding free energy between the target receptor with its ligands. Negative values indicate that the greater the bond is more stable, which means that the interaction with the ligand may hinder the performance of these receptors in causing cancer.

In general, the obtained score are between -72.217 and $66.852 \mathrm{kcal} / \mathrm{mol}$. Moldock score of ligand $2 \mathrm{HNO} 11$ is lower than doxorubicin and poloxin. Doxorubicin, known by its trade name Adriamycin or liposomeencapsulated form known as hydroxydaunorubicin with trade name Doxil is a drug used in cancer chemotherapy and derived by chemical semi synthesis from a bacterial species [13]. Poloxin is a proven active compound as inhibitors of Plk1. By blocking PBD binding to its recognition motif, poloxin disrupted the human Plk1 sub-cellular localization and eventually arrested the cell cycle $[13,14]$.

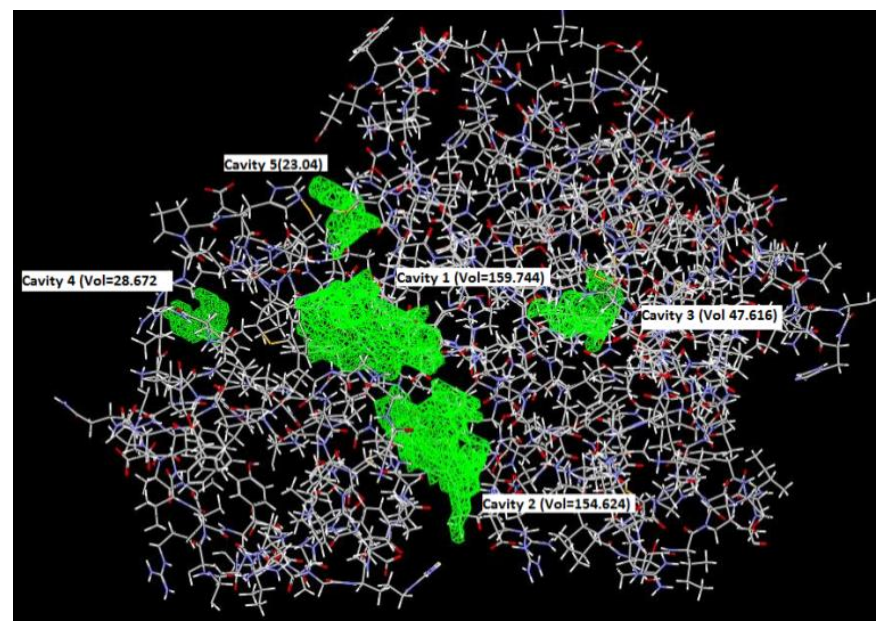

Figure 1. The five cavity MVD-detected cavities in Plk1, and their calculated volumes (in $\AA$ ) (PDB ID 3THB [10], detected cavity green, carbon atoms grey, oxygen atoms red, nitrogen atoms blue).

Moldock scores of compound HNO11, Doxorubicin and Poloxin are $-153,725,-121.436$, and $-112.865 \mathrm{kcal} / \mathrm{mol}$, respectively. Modified naphthoquinone compounds have moldock score better than the original compound (2HN). Several modified naphthoquinone compounds (HNO9, HNO10 and HNO8), even have the best score better than doxorubicin and poloxin. This results indicates that the above mentioned molecules are predicted to be anticancer drug candidate

The superposition of benzolactam, as observed in the cavity of the crystallographic structure of Plk1 and the best conformation obtained theoritically for naphthoquinone derivated are shown in Figure 2. The result suggests that the software reproduced the appropriate conformation of benzolactam inside its binding site in the Plk1.

Table 1. Docking parameters $(\mathrm{kcal} / \mathrm{mol})$ in active site of polo like kinase 1 receptor

\begin{tabular}{|c|c|c|c|}
\hline No. & Ligand & MolDock Score & Re-rank Score \\
\hline 1 & Benzolaktam & -166.852 & -135.154 \\
\hline 2 & 2HNO-11 & -153.725 & -127.115 \\
\hline 3 & 2HNO-9 & -148.028 & -119.129 \\
\hline 4 & $2 \mathrm{HNO}-10$ & -140.683 & -119.203 \\
\hline 5 & $2 \mathrm{HNO}-8$ & -140.456 & -123.051 \\
\hline 6 & Doxorubicin & -121.436 & -97.944 \\
\hline 7 & $2 \mathrm{HNO}-3$ & -120.829 & -105.711 \\
\hline 8 & $2 \mathrm{HNO}-5$ & -120.131 & -106.425 \\
\hline 9 & 2HNO-4 & -118.352 & -93.298 \\
\hline 10 & 2HNO-15 & -114.334 & -100.103 \\
\hline 11 & Poloxin & -112.865 & -94.8336 \\
\hline 12 & 2HNO-14 & -112.792 & -94.109 \\
\hline 13 & 2HNO-18 & -111.891 & -95.098 \\
\hline 14 & 2HNO-2 & -111.748 & -98.112 \\
\hline 15 & 2HNO-16 & -111.502 & -100.260 \\
\hline 16 & $2 \mathrm{HNO}-13$ & -107.674 & -92.548 \\
\hline 17 & $2 \mathrm{HNO}-12$ & -101.318 & -90.838 \\
\hline 18 & 2HNO-6 & -92.269 & -81.117 \\
\hline 19 & $2 \mathrm{HNO}-17$ & -84.254 & -75.370 \\
\hline 20 & 2HNO-7 & -74.531 & -71.983 \\
\hline 21 & $2 \mathrm{HN}$ & -72.217 & -67.197 \\
\hline 22 & 2HNO-1 & -72.217 & -67.197 \\
\hline
\end{tabular}

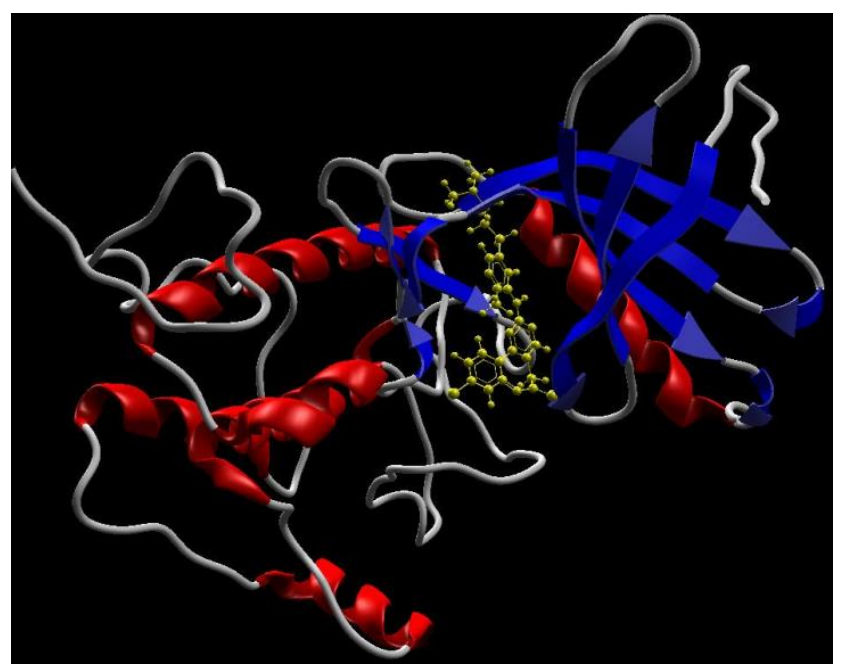

Figure 2. Structural cartoon of pololike kinase 1 (PDB ID 3THB [10], the $\alpha$ helices and $\beta$ strands are represented as coils (red) and arrows (blue), respectively. Benzolactam is represented in ball and stick (yellow). Model prepared using MolDock [9]. 


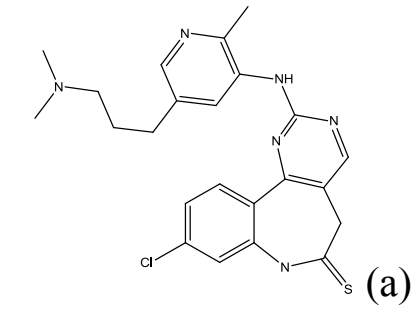<smiles>COc1cccc2c1C(=O)c1c(O)c3c(c(O)c1C2=O)C[C@@](O)(C(=O)O)C[C@@H]3[C@H]1C[C@H](N)[C@@H](O)[C@@H](C)O1</smiles>

(b)<smiles>C=c1cc(C)c(C(C)C)cc1=CC=CC(=O)c1ccccc1C</smiles>

Figure 3. Structure of (a) Benzolaktam, (b) Doxorubicin, (c) Poloxin

Binding between active site polo like kinase 1 and benzolactam, 2HNO11, Doxorubicine Poloxine on Cys 67, Arg 136, Cys 133 and Asp 194 amino acids showed in Figure 3 and Table 3, it showed the formation of hydrogen bonds of polo like kinase-1 amino acids.

The most of amino acid residues in hydrophobic active site were involved in affinity hydrophobic bonding interactions of ligands (Figure 4). This figure shows hydrophobic bonding between benzolactam, 2HNO11, Doxorubicin and Poloxine with polo like kinase 1 receptor.

The structure of benzolactam, 2HNO11 and doxorubicin was non polar, so this gave advantage to hydrophobic for binding inside chains as receptor active site. The naphthoquinone derivate (2HNO11) has similar role binding with doxorubicin that has been proven as anticancer agent and occupied in cavity 2 of polo like kinase 1 receptor toward hydrophobic binding with amine group and lacton group.

The naphthoquinone derivate has similar role binding with doxorubicin, but it has not similar role binding with benzolactam and occupied in cavity 2 of polo like kinase 1 receptor toward hydrophobic binding with sulphur and amine group. Sulphur was important for binding on the other side 2HNO11, Doxorubicin and Poloxin cannot bind well in active site of polo like kinase 1 because they didn't have sulphur. The docking studies of the naphthoquinone derivatives in the active site of polo like kinase 1 receptor showed that the Moldock Score and Re-rank score were lower than doxorubicin and poloxin. Decreasing interaction has an effect on the result of decreasing affinity binding between ligand-receptor. It is still need to know the correlation between docking score and activities of naphthoquinone derivate, doxorubicin and poloxin.

Table 2. Structure of $2 \mathrm{HN}$ derivatives

\begin{tabular}{|c|c|c|c|}
\hline Structure & & & \\
\hline Compound & $\mathrm{X} 1$ & $\mathbf{X} 2$ & $\mathbf{X 3}$ \\
\hline $2 \mathrm{HN}$ & $\mathrm{O}$ & $\mathrm{O}$ & $\mathrm{OH}$ \\
\hline 2HNO-1 & $\mathrm{O}$ & $\mathrm{NOH}$ & $\mathrm{OH}$ \\
\hline 2HNO-2 & $\mathrm{O}$ & & $\mathrm{OH}$ \\
\hline 2HNO-3 & $\mathrm{O}$ & & $\mathrm{OH}$ \\
\hline $2 \mathrm{HNO}-4$ & $\mathrm{O}$ & & $\mathrm{OH}$ \\
\hline 2HNO-5 & $\mathrm{O}$ & & $\mathrm{OH}$ \\
\hline $2 H N O-6$ & $\mathrm{O}$ & & $\mathrm{OH}$ \\
\hline $2 \mathrm{HNO}-7$ & $\mathrm{NOH}$ & $\mathrm{NOH}$ & $\mathrm{OH}$ \\
\hline $2 \mathrm{HNO}-8$ & & & $\mathrm{OH}$ \\
\hline 2HNO-9 & & & $\mathrm{OH}$ \\
\hline 2HNO-10 & & & $\mathrm{OH}$ \\
\hline 2HNO-11 & & & $\mathrm{OH}$ \\
\hline 2HNO-12 & & & $\mathrm{OH}$ \\
\hline 2HNO-13 & $\mathrm{O}$ & $\mathrm{O}$ & \\
\hline 2HNO-14 & $\mathrm{O}$ & $\mathrm{O}$ & \\
\hline 2HNO-15 & $\mathrm{O}$ & $\mathrm{O}$ & \\
\hline 2HNO-16 & $\mathrm{O}$ & $\mathrm{O}$ & \\
\hline 2HNO-17 & $\mathrm{O}$ & $\mathrm{O}$ & \\
\hline 2HNO-18 & $\mathrm{O}$ & $\mathrm{NOH}$ & \\
\hline 2HNO-19 & $\mathrm{O}$ & $\mathrm{O}$ & \\
\hline
\end{tabular}


Table 3. Hydrogen bonding interactions between benzolactam, 2HNO11, Doxorubicin and Poloxin with polo like kinase 1 receptor

\begin{tabular}{lcccc}
\hline & Cys 67 & Arg 136 & Cys 133 & Asp 194 \\
\hline Benzolactam & - & - & + & + \\
2HNO11 & - & + & - & - \\
Doxorubicin & - & + & + & - \\
Poloxin & + & - & + & - \\
\hline
\end{tabular}

Table 4. Physicochemical parameters of benzolactam, 2HNO11, doxorubicin and poloxin

\begin{tabular}{lcrrr}
\hline Ligand & Log P & Clog P & MR & CMR \\
\hline Benzolactam & 2 & 2.960 & 78 & 7.6116 \\
2HNO11 & 0 & -0.666 & 136 & 13.1721 \\
Doxorubicin & 3 & 3.606 & - & 8.7712 \\
Poloxin & 6 & 7.212 & - & 12.4348 \\
\hline
\end{tabular}

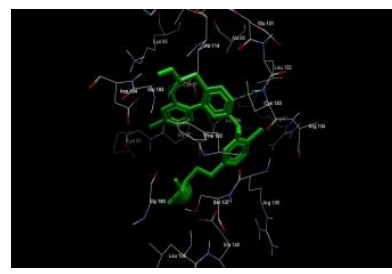

(a)

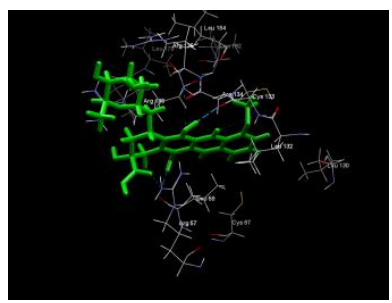

(c)

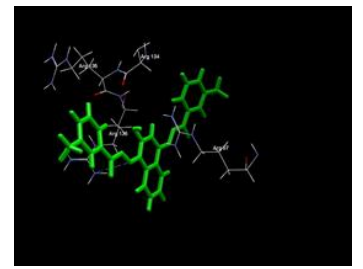

(b)

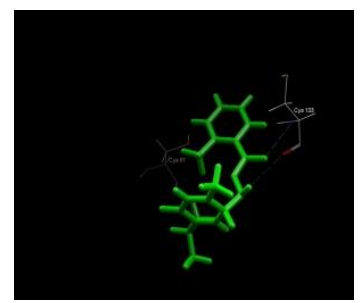

(d)
Figure 4. Hydrogen bonding interactions between polo like kinase 1 receptor and benzolactam (a), 2HNO11 (b), Doxorubicine (c), and Poloxin (d).

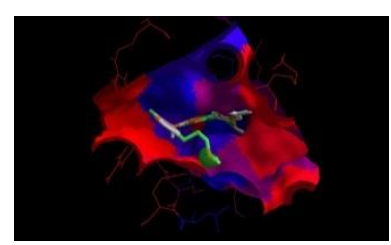

(a)

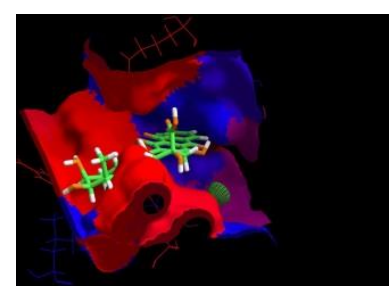

(c)

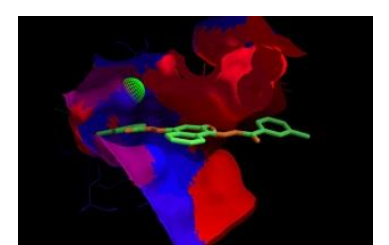

(b)

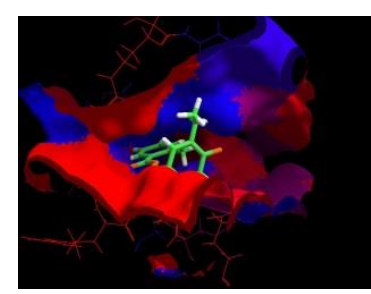

(d)
Figure 5. Hydrophobic bonding interactions between polo like kinase 1 receptor and benzolactam (a), 2HNO11 (b), Doxorubicine (c) \& Poloxin (d).

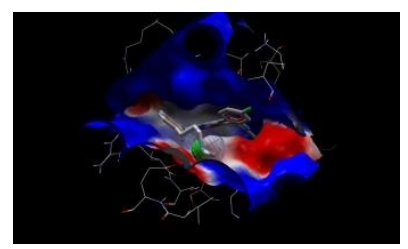

(a)

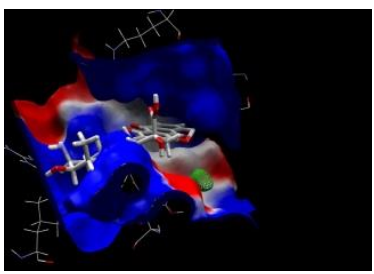

(c)

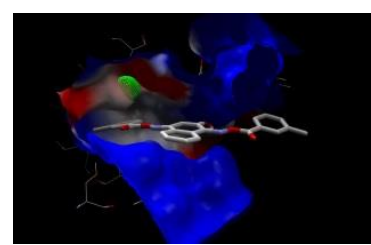

(b)

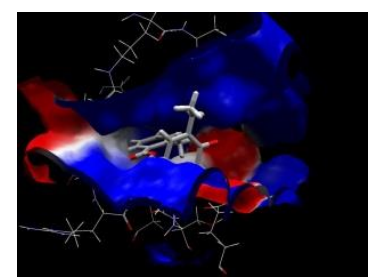

(d)
Figure 6. Electrostatic bonding interactions between polo like kinase 1 receptor and benzolactam (a), 2HNO11 (b), doxorubicine (c), and poloxin (d).

\section{Conclusions}

The results of this study showed that naphthoquinone derivate (2HNO11) was better bound into polo line kinase 1 receptor as antimytotic agent than doxorubicin and poloxin. The Moldock score and Rerankscore of this compound were -127.115 and -153.725 , respectively, lower than those of doxorubicin and poloxin and hence can be predicted as potential ligand of polo kinase 1 .

\section{Acknowledgement}

Author is thankful to Dr. Bambang Marwoto, Apt., MEng, Ir. Bambang Srijanto, Dr. Prasetyawan Yunianto, MP and Firdayani, M.Farm for their encouragement, permission and support in computational/software facilities at the Laboratory of Pharmaceutical and Medical Technology, Center of Pharmaceutical and Medical Technology, Agency for Assessment and Application of Technology.

\section{References}

[1] R.P. Verma and C. Hanch, Elucidation of structureactivity relationship for 2-or- 6- substituted- 5-8dimethoxy-1,4-naphthoquinones, Bioorganic and Medicinal Chemistry, 12, 2004, 5997-6009.

[2] R. Verma, Anti-Cancer Activities of 1,4Naphthoquinones: A QSAR Study, Anti-Cancer Agents in Medicinal Chemistry, 6, 2006, 489-499.

[3] K.V. Tandon, R.V. Singh, and D.B. Yadav, Synthesis and evaluation of novel 1,4-naphthoquinone derivatives as antiviral, antifungal and anticancer agents, Bioorganic and Medicinal Chemistry Letters, 14, 2004, 2901-2904.

[4] T. Kayashima, M. Mori, H. Yoshida, and Y. Mizushina, 1,4-Naphthoquinone is a potent inhibitor of human cancer cell growth and angiogenesis, Cancer Letter, 278, 2009, 34-40.

[5] Degenhart and Lampkin, Targeting Polo-like Kinase in cancer therapy, Clin. Cancer Res., 16(2), 2010, 384389 , 
[6] K. Strebhardt and A. Ullrich, Targeting polo-like Kinase 1 for cancer therapy, Nat. Rev. Cancer, 6, 2006, 321-330.

[7] K. Stebhardt, Multifaceted polo-like kinases: drug targets and antitargets for cancer therapy, Nature Review, 9, 2010, 643-660.

[8] S. Naeem, P. Hylands, and D. Barlow, Docking studies of chlorogenic acid against aldose reductase by using Molegro Virtual Docker Software, Journal of Applied Pharmaceutical Science, 3(01), 2013, 13-020

[9] R. Thomsen and M.H., Christensen Moldock: A new technique for high-accuracy molecular docking, $\mathrm{J}$. Med. Chem., 49, 2006, 3315-3321.

[10] M.O. Duffey , T.J. Vos, R. Adams, and J. Alley, Discovery of a potent and orally bioavailable benzolactam-derived inhibitor of Polo-Like Kinase 1 (MLN0905), J. Med. Chem., 55(1), 2012, 197-208

[11] Bruinsma et al, Switching Polo-like kinase-1 on and off in time and space, Trends in biochemical Sciences, 37(12), 2012, 543-542.

[12] Brayfield, A., ed. "Doxorubicin", Martindale: The Complete Drug Reference, Pharmaceutical Press, Retrieved 15 April 2014.

[13] Y. Zhou, C. Jianhua, and P.H. Rehse, Thymoquionone and poloxin are slow-irreversible inhibitors of human Polo-like kinase 1 polo box domain, Journal of Medical College of PLA, 25, 2010, 136-142.

[14] T.J. Lansing and R.T. MCConnell, In vitro biological activity of novel small molecule inhibitor of Polo-Like Kinase I, Mol. Cancer Ther., 6(2), 2007, 450-459. 